ARTÍCULO ORIGINAL

\title{
CRÉDITOS DEL SISTEMA FINANCIERO AL SECTOR PRIVADO Y REGULACIÓN DEL MERCADO DE CRÉDITOS EN PERÚ
}

\section{FINANCIAL SYSTEM CREDITS TO THE PRIVATE SECTOR AND CREDIT MARKET REGULATION IN PERU}

\author{
Vladimir Rodriguez Cairo \\ Doctor en Derecho y Ciencia Política, Magister en Economía - Docente Principal de la Facultad de Ciencias Contables \\ Universidad Nacional Mayor de San Marcos, Lima, Perú -Email: vrodriguezc@unmsm.edu.pe (Autor Corresponsal)
}

[Recibido: 2016/08/16 Aceptado: 2016/11/16]

\section{RESUMEN}

El objetivo de la investigación es explicar por qué la libertad en el mercado de créditos incide positivamente en los créditos del sistema financiero al sector privado en el Perú, generando un aumento del stock de créditos al sector privado. A partir de dicho objetivo, se realizó un análisis descriptivo e inferencial sobre la relación entre las variables. Siendo así, se obtuvieron como principales resultados que la libertad en el mercado de créditos como componente de la regulación del mercado de créditos, influyó en el stock de créditos del sistema financiero al sector privado del país. De este modo, se concluyó que la libertad en el mercado de créditos favorece el stock de créditos y consiguientemente, contribuye a profundizar el sistema financiero. No obstante, la mayor libertad en el mercado de créditos es un aspecto esencial para aumentar los créditos al sector privado pero no es el único elemento.

\section{PALABRAS CLAVE}

Stock, créditos, Sistema financiero, sector privado, regulación, mercado, libertad de mercado.

\begin{abstract}
The objective of the research is to explain why the freedom in the credit market positively affects the loans of the financial system to the private sector in Peru, generating an increase in the stock of loans to the private sector. Based on this objective, a descriptive and inferential analysis was performed on the relationship between the variables. Thus, the main results were that the freedom in the market of credits as a component of the regulation of the market of credits, influenced in the stock of credits of the financial system to the private sector of the country. In this way, it was concluded that the freedom in the credit market favors the stock of credits and, consequently, contributes to deepening the financial system. However, greater freedom in the credit market is an essential aspect of increasing credits to the private sector, but it is not the only element.
\end{abstract}

\section{KEYWORDS}

Stock, loans, financial system, private sector, regulation, market, market freedom.

Como Citar: Rodríguez, V. (2017). Créditos del sistema financiero al sector privado y regulación del mercado de créditos en Perú. Quipukamayoc, 25(47), 9 - 18. doi: http://dx.doi.org/10.15381/quipu.v25i47.13798

\section{INTRODUCCIÓN}

El estudio examina la relación entre la libertad en el mercado de créditos y el stock de créditos del sistema financiero al sector privado en Perú. Para tal efecto, se formula la siguiente interrogante: ¿Por qué la libertad en el mercado de créditos incide positivamente en los créditos del sistema financiero al sector privado, generando un incremento del nivel de stock de los créditos al sector privado? Es así que la hipótesis que responde a la pregunta es: "A mayor libertad en el mercado de créditos existe mayor stock de créditos del sistema financiero al sector privado en Perú en el período 2000-2014". Para tal efecto, se exponen los fundamentos teóricos del orden espontáneo y el orden construido (Hayek, 2014). Desde tal perspectiva, también se sustenta la teoría institucional de la economía para enfatizar la importancia de las reglas de juego en el sistema económico (North, 1993).

Asimismo, se fundamenta la teoría del crédito (Mises, 2012) y el mercado de crédito (Varian, 2006). Un aspecto importante en el presente estudio, es el análisis de los datos del grado de libertad en el mercado de créditos como componente de la regulación del Economic Freedom of the World 2016 Annual Report publicado por Fraser Institute de Canadá.

Bajo tal premisa, el estudio recurre al enfoque cuantitativo, por ser probatorio a través de mediciones estadísticas. Por cierto, la principal limitación de la investigación es el número

( ) Los autores. Este artículo es publicado por la Revista Quipukamayoc, Universidad Nacional Mayor de San Marcos. Este es un artículo de acceso abierto, distribuido bajo los términos de la Licencia Creative Commons Atribución-NoComercial-Compartirlgual 4.0 Internacional.(http://creativecommons.org/licenses/by-nc-sa/4.0/), que permite el uso no comercial, distribución y reproducción en cualquier medio, siempre que la obra original sea debidamente citadas. 
de observaciones disponibles, el cual restringe el uso de análisis estadísticos más avanzados.

En lo esencial, la mayoría de estudios analizan la relación entre libertad económica y crecimiento de diversos países, a diferencia del presente estudio que se orienta a conocer la relación particular que existe entre la regulación a través de la libertad en el mercado de créditos y el nivel de stock de créditos del sistema financiero al sector privado en nuestro país. Dentro de este contexto, la libertad en el mercado de créditos implica una mayor profundidad del sistema financiero.

El objetivo de la investigación es explicar por qué la libertad en el mercado de créditos incide positivamente en los créditos del sistema financiero al sector privado en el Perú, generando un aumento del stock de créditos al sector privado.

Tabla 1.

Dos fuentes de orden: espontáneo (evolutivo) y construido (artificial) Fuente: Elaboración Propia

\begin{tabular}{|c|c|}
\hline Orden Espontáneo & Orden Construido \\
\hline Orden que se autogenera o endógeno & Orden que se construye o exógeno \\
\hline Formado por la evolución & $\begin{array}{l}\text { Creado artificialmente a través del diseño } \\
\text { humano }\end{array}$ \\
\hline $\begin{array}{l}\text { Proviene de acciones humanas no inten- } \\
\text { cionadas encaminadas a otros fines }\end{array}$ & $\begin{array}{l}\text { Proviene de planes intencionados (deli- } \\
\text { berados) }\end{array}$ \\
\hline $\begin{array}{l}\text { Carece de finalidad concreta (cuyo conte- } \\
\text { nido particular no es conocido o previsto } \\
\text { por nadie) }\end{array}$ & $\begin{array}{l}\text { Tiene fines concretos (mandados especí- } \\
\text { ficos) }\end{array}$ \\
\hline $\begin{array}{l}\text { Sustentado por el racionalismo evolu- } \\
\text { cionista (reconoce la abstracción como } \\
\text { único instrumento con el que nuestra } \\
\text { mente puede tratar una realidad cuya } \\
\text { plena comprensión se le escapa) }\end{array}$ & $\begin{array}{l}\text { Sustentado en el racionalismo cons- } \\
\text { tructivista (no reconoce los conceptos } \\
\text { abstractos son un medio para vencer la } \\
\text { complejidad de lo concreto) }\end{array}$ \\
\hline $\begin{array}{l}\text { Instituciones de mayor importancia: el } \\
\text { lenguaje, la sociedad, la costumbre, la } \\
\text { moral, la moneda, el trueque, el mercado, } \\
\text { la competencia, el liberalismo, el sistema } \\
\text { de precios y el Derecho. }\end{array}$ & $\begin{array}{l}\text { Concibe todas las instituciones como } \\
\text { resultados de planes deliberados: la fa- } \\
\text { milia, el gobierno, una organización, una } \\
\text { empresa, el ejército y la legislación. }\end{array}$ \\
\hline $\begin{array}{l}\text { Griegos: utilizaron el término "cosmos" } \\
\text { para el orden formado por evolución. }\end{array}$ & $\begin{array}{l}\text { Griegos: utilizaron el término "taxis" para } \\
\text { el orden creado artificialmente. }\end{array}$ \\
\hline
\end{tabular}

En base a la teoría del Orden Espontáneo (Derecho) y Orden Construido (legislación), Hayek (2014) precisa que:

(...) Tales órdenes (construidos o taxis) son órdenes relativamente simples (...); suelen ser concretos (...) que su existencia puede percibirse intuitivamente por simple observación; y, finalmente, al haber sido construidos deliberadamente, sirven invariablemente a los fines de su creador. Ninguna de estas características pertenece necesariamente a un orden espontáneo o cosmos. Su grado de complejidad no está limitado a lo que una mente humana pueda dominar. Su existencia no necesita manifestarse a nuestros sentidos, sino que puede basarse simplemente en relaciones abstractas que nosotros sólo podemos reconstruir

mentalmente. Y al no haber sido construidos deliberadamente, no se puede legítimamente decir que tengan un objetivo particular ( $\mathrm{p}$. 61-62).

La idea primordial de Hayek (2014) es que el Orden Espontáneo, es el sistema que se autogenera de manera evolutiva por las acciones humanas no intencionadas, es un orden que no ha sido creado artificialmente (deliberadamente) a través del diseño humano, por tanto, no es susceptible de conocimiento completo por parte de los individuos. Argumenta que el orden espontáneo se descubre o se desarrolla a través de un proceso de prueba y error, en el que los grupos sociales irán adaptando o reajustando sus acciones individuales sobre la base de los nuevos elementos que van apareciendo (ya sean conocimientos, valores, costumbres, normas, precios, etc.), de tal manera que solo aquellos elementos que faciliten el intercambio o la supervivencia de dichos grupos tenderán a prevalecer sobre aquellos elementos menos eficaces.

A continuación, se puede apreciar las principales diferencias entre el orden espontáneo que proviene las acciones humanas individuales (carece de finalidad concreta) y el orden construido que es el resultado del diseño humano (tiene fines concretos).

De lo expresado anteriormente, se desprende que dentro del orden espontáneo se encuentra el Derecho (producto del racionalismo evolutivo), y dentro del orden construido, que es el resultado del diseño humano, se encuentra la legislación (producto del racionalismo constructivista). Similar al lenguaje, el trueque, la moneda, el mercado, la competencia, la costumbre, el sistema de precios; lo mismo ocurrió con el Derecho. En efecto, el common law (Derecho anglosajón) fue producto de decisiones judiciales que fueron elaborando principios (los 
cuales se iban modificando), se basaban en costumbres y poco a poco ese Derecho fue evolucionando, pero no como consecuencia de una autoridad especial sino por la acción de miles de personas litigando y miles de jueces resolviendo los litigios.

Las formas en que las instituciones y los cambios institucionales afectan la economía. Define las instituciones como: "Las reglas de juego de una sociedad o más formalmente, son las limitaciones ideadas por el hombre que dan forma a la interacción humana. Por consiguiente, estructuran incentivos en el intercambio humano, sea político, social o económico. El cambio institucional conforma el modo en que las sociedades evolucionan a lo largo del tiempo, por lo cual es la clave para entender el cambio histórico" (North, 1993, p. 13).

Las instituciones se componen de: 1) reglas formales, 2) reglas informales, 3) cumplimiento de las reglas (ejecución obligatoria). Ahora bien, el marco institucional, abarca la estructura de la organización política de un país que especifica nuestra manera de elaborar y agregar decisiones políticas (conformado por los órganos del Estado en sus distintos niveles) y la estructura social que define los incentivos informales de la economía (basados esencialmente en la cultura ). Bajo tal premisa, el sistema político (integrado por los diversos órganos del Estado dentro de su respectivo ámbito: nacional, regional o local) conduce a la selección de reglas formales, las cuales conjuntamente con las reglas informales (que provienen de la información transmitida socialmente y son parte de la herencia o cultura) determinan el marco institucional y contribuyen a garantizar el buen desempeño económico.

Aunque las instituciones formales pueden cambiar de la noche a la mañana como resultado de decisiones políticas o judiciales, las instituciones informales encajadas en costumbres, tradiciones y códigos de conducta son mucho más resistentes o impenetrables a las políticas deliberadas. No obstante ello, el Estado a través de todos sus órganos interviene en el mercado ante las diversas fallas que presenta éste, dentro de las competencias $\mathrm{y}$ funciones asignadas por las normas legales (Constitución, Leyes, Decretos Supremos, Resoluciones, entre otras). La regulación forma parte del proceso político.

Siendo así, el marco institucional, conformado por reglas formales e informales, permite establecer incentivos políticos, económicos, sociales, entre otros; los cuales fomentan intercambios eficientes y la productividad de la economía. En ese propósito, cumplen un rol fundamental las organizaciones (políticas, económicas, sociales, sociedad civil en su conjunto) como agentes del cambio institucional.

En efecto, se deben establecer incentivos económicos, entre los cuales destacan garantizar los derechos de propiedad, asegurar el cumplimiento de contratos, reducir los costos de transacción y atenuar los problemas de

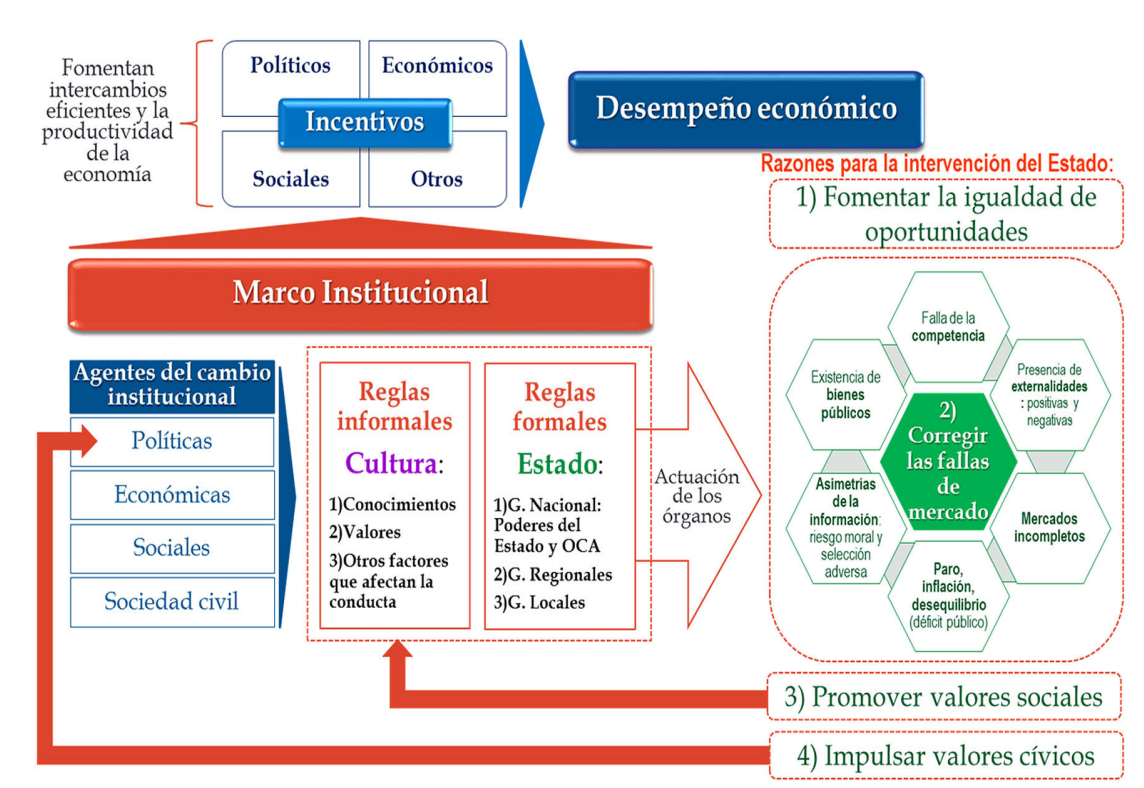

Figura 1. Marco institucional y su implicancia en el desempeño económico

Fuente: Elaboración Propia información para el buen desempeño económico de un país. En esa misma línea, es importante contar con incentivos políticos como garantizar la estabilidad política, asegurar el régimen democrático, fortalecer las organizaciones políticas y garantizar un sistema político que promueva el Estado de Derecho. Asimismo, como incentivos sociales se debe promover la igualdad, garantizar la justicia y el bienestar. Del mismo modo, resulta trascendente fomentar los valores sociales. En la siguiente figura se aprecia la interacción del Estado y la cultura como fundamentos esenciales del marco institucional que define los incentivos políticos, económicos, sociales; los cuales definitivamente contribuyen al desempeño económico. Evidentemente, un elemento muy relevante de este marco institucional es la Constitución Política, la cual establece las "grandes reglas de juego" de un país en materia política, económica y social para un cierto tiempo de su desarrollo, y sobre todo, se caracteriza por orientar hacia la consecución de determinados fines. El régimen económico, al ser un componente fundamental de la Constitución Política, define los incentivos económicos de una 
sociedad libre orientados a fortalecer el intercambio eficiente en el mercado, y consiguientemente, a garantizar el buen desempeño económico.

Según la teoría del crédito propuesta por Mises (2012), argumenta que:

la actividad de los bancos como negociadores de crédito debe crearse una conexión orgánica entre el activo y el pasivo de sus transacciones (...), la fecha en que vencen las obligaciones del banco no debe preceder a la fecha en que las obligaciones frente a él puedan hacerse efectivas. Solamente así puede evitarse el riesgo de insolvencia y la certeza de que queda un riesgo; pero que la concesión imprudente de créditos puede ser de consecuencias tan ruinosas para un banco como para cualquier otro comerciante. Esto se produce por la estructura jurídica de su negocio; no existe relación jurídica entre sus transacciones de activo y sus transacciones de pasivo, y su obligación de devolver el dinero tomado a préstamo no está afectada por el destino de sus inversiones; la obligación continúa incluso si las inversiones han resultado pérdidas irreparables. Pero es precisamente la existencia de este riesgo lo que hace provechoso para el banquero el representar el papel de intermediario entre el que da un crédito y el que los recibe. Y que de la aceptación de este riesgo es de donde el banco obtiene sus beneficios y sus pérdidas(p. 297-298).

Ante lo sostenido por Mises (2012), es importante resaltar la precisión que realiza acerca de la existencia del riesgo en las operaciones de los bancos, la cual justamente resulta provechoso para el banquero, quien al aceptar el riesgo puede obtener beneficios o pérdidas.
El mercado de créditos es "la cantidad de créditos que se solicitan y se conceden en una economía depende en gran medida del tipo de interés que se cobre" (Varian, 2006 , p. 307). Éste es el precio del mercado de créditos.

Sea $\mathrm{D}(\mathrm{r})$ la demanda de crédito de los prestatarios y $\mathrm{S}(\mathrm{r})$ la oferta de crédito de los prestamistas. El tipo de interés de equilibrio, $r^{\star}$, es aquel al que la demanda es igual a la oferta: $\mathrm{D}\left(\mathrm{r}^{\star}\right)=$ $\mathrm{S}\left(\mathrm{r}^{*}\right)$ Supongamos que introducimos los impuestos en este modelo. ¿Qué ocurrirá con el tipo de interés de equilibrio? En la mayoría de los países, los intereses que rinden los préstamos están sujetos al impuesto sobre la renta. Si todo el mundo se encuentra en el mismo intervalo impositivo t, el tipo de interés una vez deducidos los impuestos que reciben los prestamistas es $(1-t) r$. Por lo tanto, la oferta de crédito, que depende de este tipo de interés, es $S((1-t) r)$.

El Índice de regulación del mercado de créditos, es un término regulación financiera, se utiliza comúnmente para referirse a todas las normas que tengan un contenido financiero y se apoya en el Derecho, la Economía y la Ciencia Política con la finalidad de entender cómo las reglas de juego influencian la conducta de los agentes que participan en los mercados financieros.

El Reporte Anual del Economic Freedom of the World (EFW) 2016 publicado por Fraser Institute de Canadá, el Índice de Libertad Económica en el Mundo mide el grado en que las políticas y las instituciones de los países apoyan la libertad económica. Las piedras angulares de la libertad económica son la elección personal, el intercambio voluntario, la libertad para entrar en los mercados y competir y la seguridad de la persona y la propiedad privada. Es así que el Índice de Libertad Económica se compone de cinco áreas generales (escala de 1 a 10): I) Tamaño del gobierno, II) Estructura legal, III) Moneda sana, IV) Libertad de comercio internacional, V) Regulación.

El presente estudio aborda la temática del área V), esto es, la regulación (Rodríguez, 2016).

En ese sentido, tal como se sostiene en el EFW, cuando las regulaciones restringen la entrada a los mercados e interfieren con la libertad de participar en el intercambio voluntario, reducen la libertad económica. La quinta área del índice analiza las restricciones regulatorias que limitan la libertad de intercambio en los mercados de crédito, laborales y de productos. Es así que los países que utilizan un sistema de banca privada para asignar el crédito a particulares y se abstienen de controlar las tasas de interés reciben las calificaciones más altas en este indicador. Con el fin de obtener altas calificaciones en esta parte del índice, los países deben permitir que los mercados determinen los precios y abstenerse de actividades reguladoras que retarden la entrada de empresas y aumenten el costo de producción de los productos. Tal como se aprecia en la Tabla 2, se utilizan tres (3) componentes para la construcción del índice de regulación del mercado de créditos o libertad en el mercado de créditos. (Siguiente página)

Nuestra Constitución Política vigente, además de establecer la finalidad del Banco Central de Reserva del Perú - BCRP (preservar la estabilidad monetaria), también le asigna las siguientes funciones: regular la moneda y el crédito del sistema financiero, administrar las reservas internacionales a su cargo, emitir billetes y monedas e informar periódicamente al país sobre las finanzas nacionales.

Al referirnos a la crisis financiera de 2008, sostienen que el nivel bajo de la confianza ha provocado la congelación de los mercados de crédito. 
Las entidades de crédito no confían en que conseguirán cobrar. En las circunstancias actuales, los que desean gastar encuentran dificultades para obtener el crédito que necesitan y a los suministradores de bienes les cuesta obtener el capital circulante que requieren. El resultado es que los multiplicadores fiscales habituales, procedentes del incremento del gasto gubernamental o de la reducción de impuestos, serán seguramente mucho menores. (Akerlof y Shiller 2014, p. 42)

\section{MATERIAL Y MÉTODOS}

El estudio realizado fue descriptivo y correlacional. Se analizó la evolución del stock de créditos del sistema financiero al sector privado para Perú. Igualmente, se comparó la evolución del grado de la regulación del mercado de créditos (grado de libertad en el mercado de créditos) entre los países miembros de la Alianza del Pacífico (Perú, Chile, Colombia y México). Del mismo modo, se describieron los resultados de la libertad en el mercado de créditos.

Presentando un diseño no experimental pues no se manipulan variables, es decir, no se tiene el control directo de la variable independiente.

Teniendo un enfoque cuantitativo por ser probatorio a través de mediciones estadísticas.

Y tomando como unidad de análisis a los datos del stock de los créditos del sistema financiero al sector privado y el grado de libertad en el mercado de créditos en Perú para el período 2000-2014.

El Tratamiento de los datos y análisis de la información se realizaron a partir de los datos obtenidos del Índice de Regulación del Mercado de Créditos Rodríguez (2014) elaborado por el Instituto Fraser y los datos de los créditos del sistema financiero al sec-

Tabla 2.

Componentes y subcomponentes de la regulación

Fuente: Gwartney, J. - Lawson R. - Hall J. (2016) Economic Freedom of the World 2016 Annual Report. Fraser Institute

\begin{tabular}{ll}
\multicolumn{2}{c}{ V. Regulación } \\
A. Regulación del mercado de créditos & C. Regulación de empresas \\
\hline (i) Propiedad de los bancos & (i) Requerimientos administrativos \\
\hline (ii) Crédito del sector privado & (ii) Costos de la burocracia \\
\hline (iii) Control sobre las tasas de interés / tasa & (iii) Creación de una empresa \\
de interés reales negativas & \\
\hline B. Regulación del mercado de trabajo & (iv) Pagos extra / sobornos / favori- \\
\hline (i) Reglamentos de contratación y salario & (v) Restricciones de licencia \\
mínimo & \\
\hline (ii) Reglamentos de contratación y despido & (vi) Costo del cumplimiento tributario \\
\hline (iii) Negociación colectiva centralizada & \\
\hline (iv) Reglamentos sobre las horas de trabajo & \\
\hline (v) Costo del despido de un trabajador & \\
\hline (vi) Servicio militar obligatorio & \\
\hline
\end{tabular}

tor privado, se aplicaron las siguientes técnicas estadísticas y métodos de estimación:

- Análisis descriptivo para determinar la relación entre el índice de regulación del mercado de créditos y el stock de los créditos del sistema financiero al sector privado. Asimismo, se determina la composición del índice de regulación del mercado de créditos para Perú. También se compara esos datos entre los países miembros de la Alianza del Pacífico.

- Análisis inferencial a través de pruebas estadísticas para verificar la existencia y sentido de relación entre las dos variables de interés. Dentro del análisis inferencial, cabe precisar que la principal limitante del estudio fue la disponibilidad de datos, lo cual restringió el uso de análisis estadísticos más avanzados. Dado que se contó con una serie anual de corta duración, tampoco se utilizaron variables que podrían haber enriquecido el modelo para no perder grados de libertad. Al mismo tiempo, si bien los datos corresponden a series de tiempo, dado que conciernen al seguimiento de las variables a lo largo del tiempo, se optó por tratarlos como un corte transversal, el cual opera sobre la base que las observaciones son independientes del tiempo debido a que las estimaciones como una serie de tiempo de corta duración llevaría a inferencias que afectan la interpretación de los resultados. Por tal motivo, a efectos de comprobar la hipótesis se recurrieron a dos aproximaciones: el coeficiente de correlación de Spearman y una estimación mediante Mínimos Cuadrados Ordinarios (MCO), utilizando el programa estadístico Stata.

Además, se empleó el coeficiente de correlación de Spearman es una prueba estadística no paramétrica que sirve para verificar la relación entre dos variables. A diferencia del coeficiente de correlación de Pearson, esta prueba no requiere asumir normalidad en la 
distribución de las variables.

Con ella se verificó la existencia y sentido de relación entre las dos variables de interés mediante el coeficiente de correlación de Spearman, calculado de la siguiente manera:

$$
r_{s}=1-\frac{6 \sum_{i=1}^{n}\left(d_{i}\right)_{i}^{2}}{n\left(n^{2}-1\right)}
$$

En donde:

$d_{i}$ es la diferencia de rangos en el par $i$ $\mathrm{n}$ es el número de pares

Cuando el número de observaciones es mayor a 20 , se puede utilizar una aproximación a la distribución $\mathrm{t}$ - Student

$$
\mathrm{T}=\mathrm{r} /\left(\left(1-\mathrm{r}^{2}\right) /(\mathrm{n}-2)\right)^{0,5}
$$

De manera que $r \_s \in[-1,1]$ y -1 se refiere a una relación perfectamente negativa, 0 a que no existe relación y 1 a una relación perfectamente positiva. Además, el estadístico muestra el nivel de significancia de la relación. Así, si Prob > $|\mathrm{t}|$ es menor al valor de significancia elegido $(0,01$; $0,05$ o 0,1$)$ se rechaza la hipótesis nula de independencia entre las variables, por lo que se concluye que existe una relación estadísticamente significativa entre las variables. Asimismo, como el análisis descriptivo y gráfico de las series muestran indicios de un cambio de comportamiento, específicamente entre los periodos 2000-2004 y 2005-2014, se evaluó la correlación tanto en el periodo 20002014 como para el 2005-2014. Ello de-

Tabla 3.

Descripción e interpretación de estadísticos

Fuente: Cameron, C. - Trivedi, P. (2009) World 2016 Annual Report. Fraser Institute / Elaboración propia

\begin{tabular}{lll}
\multicolumn{1}{c}{ Estadísticos } & \multicolumn{1}{c}{ Descripción } & \multicolumn{1}{c}{ Interpretación } \\
Prob $>$ F & $\begin{array}{l}\text { Señala que las variables utilizadas } \\
\text { mejoran la bondad de ajuste del } \\
\text { modelo. }\end{array}$ & $\begin{array}{l}\text { El modelo es mejor si este } \\
\text { valor tiende a 0 y es menor } \\
\text { a 0.05 }\end{array}$ \\
\hline R2 & $\begin{array}{l}\text { Señala la bondad de ajuste del } \\
\text { modelo. }\end{array}$ & $\begin{array}{l}\text { El modelo es mejor si } \\
\text { tiende a 1 }\end{array}$ \\
\hline
\end{tabular}

bido a que un cambio en el comportamiento durante el periodo de estudio podría significar la existencia de una relación no lineal durante ese tramo.

Como complemento al análisis de correlación, se buscó estimar una regresión que relacione ambas variables de interés, pero que también considere la existencia de un cambio de comportamiento durante el periodo 2000-2005. Para ello, se estimó mediante un modelo MCO con errores estándar robustos la siguiente función:

$$
\mathrm{Y}=\mathrm{F}(\mathrm{X}, \mathrm{D})
$$

En donde:

Y es el stock de los créditos del sistema financiero al sector privado

$\mathrm{X}$ es el grado de libertad en el mercado de créditos en Perú para el período 2000-2014.

$\mathrm{D}$ es una variable dicotómica que toma el valor de 1 durante el periodo 2000-2005, y 0 en caso contrario.

Así, el modelo a estimar fue el siguiente:

$$
\mathrm{Y}=\alpha+\beta \mathrm{X}+\gamma \mathrm{D}+\boldsymbol{\varepsilon}
$$

En donde:

$\beta$ es el coeficiente asociado a $\mathrm{X}$ $\gamma$ es el coeficiente asociado a D $\varepsilon$ es el término de error aleatorio.

Por otro lado, la idoneidad del modelo fue evaluada por medio de los siguientes estadísticos:
Prob $>$ F

Compara el modelo sin predictores (solo con el intercepto) con el modelo especificado.

$\mathrm{R} 2$

Indica que proporción de la varianza de la variable dependiente es explicada por las variables independientes.

De la misma manera, respecto a las variables exógenas, obtener un $\mathrm{p}$-value significativo asociado al $\beta$ o $\gamma$ identifica una relación estadísticamente significativa entre las variables exógenas y endógenas. Además el valor del $\beta$ o $\gamma$ señala el sentido de la relación entre ambas variables, por lo que $\beta>0$ o $\gamma>0$ muestra una relación positiva y $\beta<0 \mathrm{o}$ $\gamma<0$ lo contrario.

\section{RESULTADOS}

Hipótesis: "A mayor libertad en el mercado de créditos existe mayor stock de créditos del sistema financiero al sector privado en Perú en el período 2000-2014".

De ahí que la Hipótesis fue evaluada, analizada e interpretada tomando en cuenta las siguientes variables:

Variable endógena: stock de créditos del sistema financiero al sector privado

Variable exógena: libertad en el mercado de créditos

Análisis descriptivo de la relación entre los créditos del sistema financiero al sector privado y el índice de regulación del mercado de créditos

El Reporte Anual del Economic Freedom of The World (EFW) publicado por el Instituto Fraser es un instrumento valioso no sólo para medir la libertad económica de un país a través del tiempo, la cual toma en consideración diversos aspectos que contribuyen a explicar la libertad. También 
Tabla 4.

Perú: Créditos del sistema financiero al sector privado e Índice de regulación del mercado de créditos 2000-2014 Fuente: Fraser Institute, BCRP / Elaboración propia

\begin{tabular}{|c|c|c|c|c|c|c|c|c|c|c|c|c|c|c|c|}
\hline & 2000 & 2001 & 2002 & 2003 & 2004 & 2005 & 2006 & 2007 & 2008 & 2009 & 2010 & 2011 & 2012 & 2013 & 2014 \\
\hline $\begin{array}{l}\text { Índice de regulación } \\
\text { del mercado de } \\
\text { créditos (libertad } \\
\text { en el mercado de } \\
\text { créditos) }\end{array}$ & 9,21 & 9,20 & 9,36 & 9,32 & 9,43 & 8,84 & 8,67 & 8,45 & 8,50 & 8,42 & 9,33 & 9,33 & 9,33 & 9.67 & 9.65 \\
\hline $\begin{array}{l}\text { Propiedad de los } \\
\text { bancos }\end{array}$ & 10,00 & 10,00 & 10,00 & 10,00 & 10,00 & 8,00 & 8,00 & 8,00 & 8,00 & 8,00 & 10,00 & 10,00 & 10,00 & 10,00 & 10,00 \\
\hline $\begin{array}{l}\text { Crédito del sector } \\
\text { privado }\end{array}$ & 8,62 & 8,61 & 9,09 & 8,96 & 9,28 & 9,52 & 10,00 & 9,34 & 9,51 & 9,27 & 10,00 & 10,00 & 10,00 & 10,00 & 9.95 \\
\hline $\begin{array}{l}\text { Control sobre las } \\
\text { tasas de interés / } \\
\text { tasas de interés reales } \\
\text { negativas }\end{array}$ & 9,00 & 9,00 & 9,00 & 9,00 & 9,00 & 9,00 & 8,00 & 8,00 & 8,00 & 8,00 & 8,00 & 8,00 & 8,00 & 9,00 & 9,00 \\
\hline $\begin{array}{l}\text { Créditos del sistema } \\
\text { financiero al sector } \\
\text { privado (en millones } \\
\text { de } S / . \text { ) }\end{array}$ & 56326 & 51364 & 52531 & 52270 & 53456 & 63154 & 69434 & 88191 & 112728 & 119364 & 138873 & 166491 & 189786 & 221787 & 252936 \\
\hline
\end{tabular}

permite realizar investigaciones acerca de los efectos de los componentes de la libertad económica, dentro de los cuales se encuentra la libertad en el mercado de créditos (índice de regulación del mercado de créditos) sobre diversas variables de resultados, entre las cuales se encuentra el stock de créditos del sistema financiero al sector privado.

En la Tabla 4, se observa la evolución de las variables endógena y exógena que se utilizaron para el análisis de las pruebas estadísticas. Asimismo, se puede apreciar la evolución de los componentes del índice de regulación del mercado de créditos. Nótese que en lo referido a propiedad de los bancos, en los últimos 4 años, se alcanza el mayor grado (10), en cambio en el crédito al sector privado, si bien en el período 2010-2013 se obtuvo el mayor grado (10), en el año 2014, disminuye ligeramente $(9,95)$.

Adicionalmente, en lo referido al control de las tasas de interés, el indicador mejora para los años 2013-2014, situándose en una calificación de 9 , a diferencia de lo alcanzado en el período 2006-2012 con una calificación de 8 .
El Instituto Fraser de Canadá publica los estimados sobre la base de información con un retraso de dos años.

Como se observa en la Figura 1, el stock de los créditos del sistema financiero al sector privado y el índice de regulación del mercado de créditos siguieron un similar trayecto para el período 2000-2004 y 2010-2014, deno- tando en dichos tramos una estrecha relación entre los mismos. Sin embargo, en el período 2005-2009, mientras el índice de regulación del mercado de créditos disminuye, el stock de créditos aumenta ligeramente. En estricto, a partir del año 2009, el índice de regulación del mercado de créditos aumenta hasta alcanzar su nivel máximo en el año $2013(9,67)$, disminuyendo a

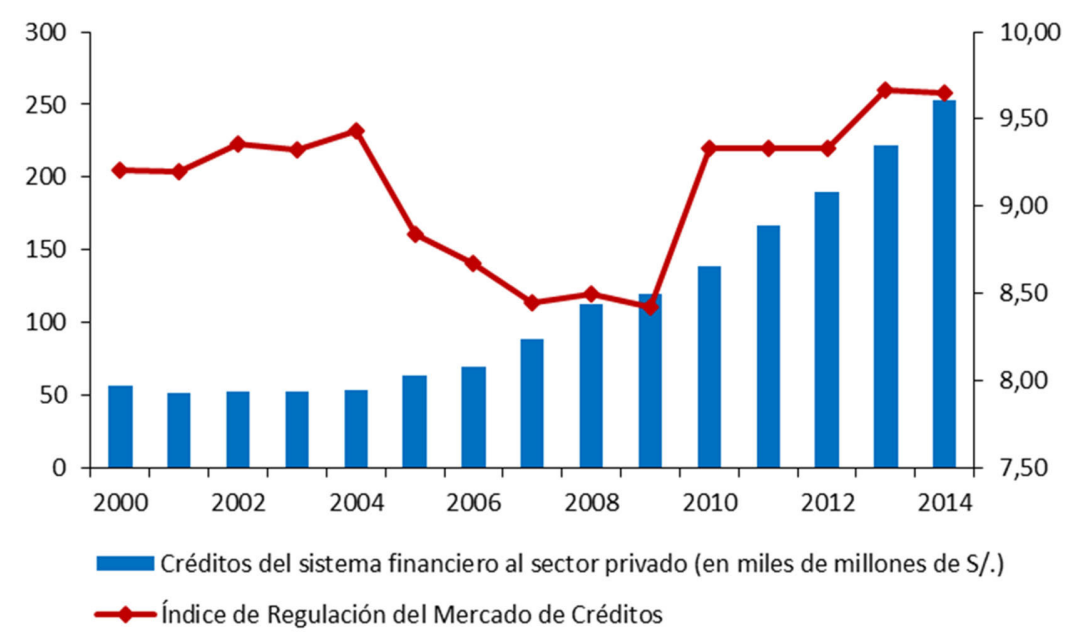

Figura 1. Perú: Evolución de los créditos del sistema financiero al sector privado e índice de regulación del mercado de créditos 2000-2014

(Expresado en miles de millones de soles)

Fuente: Fraser Institute, BCRP / Elaboración propia 
9,65 en el año 2014.

Los altos niveles obtenidos por nuestro país en el grado de libertad en el mercado de créditos implican que nuestro sistema se basa en la banca privada para asignar el crédito a particulares, tal como se puede desprender de los principios de la regulación que contempla la Ley de Bancos. Asimismo, si bien el Banco Central de Reserva del Perú (BCRP) tiene las facultades para fijar las tasas de interés máximas en el ejercicio de sus funciones y excepcionalmente puede fijar las tasas máximas y mínimas con el propósitos de regular el mercado, no controla directamente las tasas de interés del sistema financiero, razón por la cual nuestro país también se recibió las calificaciones más altas en este indicador.

\section{Análisis de la libertad en el mercado de créditos en países de la Alianza del Pacífico}

Como se observa en la Figura 2, entre los años 2000 y 2014, los indicadores de libertad en el mercado de créditos de los países miembros de la Alianza del Pacífico (Perú, Chile, Colombia y México) siguieron trayectos disímiles, siendo Chile el que presentó un comportamiento más equilibrado frente al resto de países que se caracterizaron por sus avances y retrocesos periódicos. En tanto, Perú es el país que más crece en el índice de libertad en el mercado de créditos, explicado fundamentalmente por la mayor competencia entre las empresas del sistema financiero y el ingreso de nuevos participantes junto a innovaciones financieras.

Definitivamente, el esquema actual de libre competencia del sistema financiero peruano -sin controles de tasas de interés- ha permitido brindar mejores condiciones y oportunidades a los diversos agentes económicos, con lo cual se ha dado un mayor acceso de la población al sistema financiero.

Es así que de acuerdo con Choy, Costa y Churata (2015), "La competencia en los últimos años ha implicado que las entidades financieras busquen ser más eficientes, ya que la disminución de las tasas activas implícitas ha originado una reducción del margen de ganancia, que ha sido amortiguada por menores gastos operativos". (p. 34)

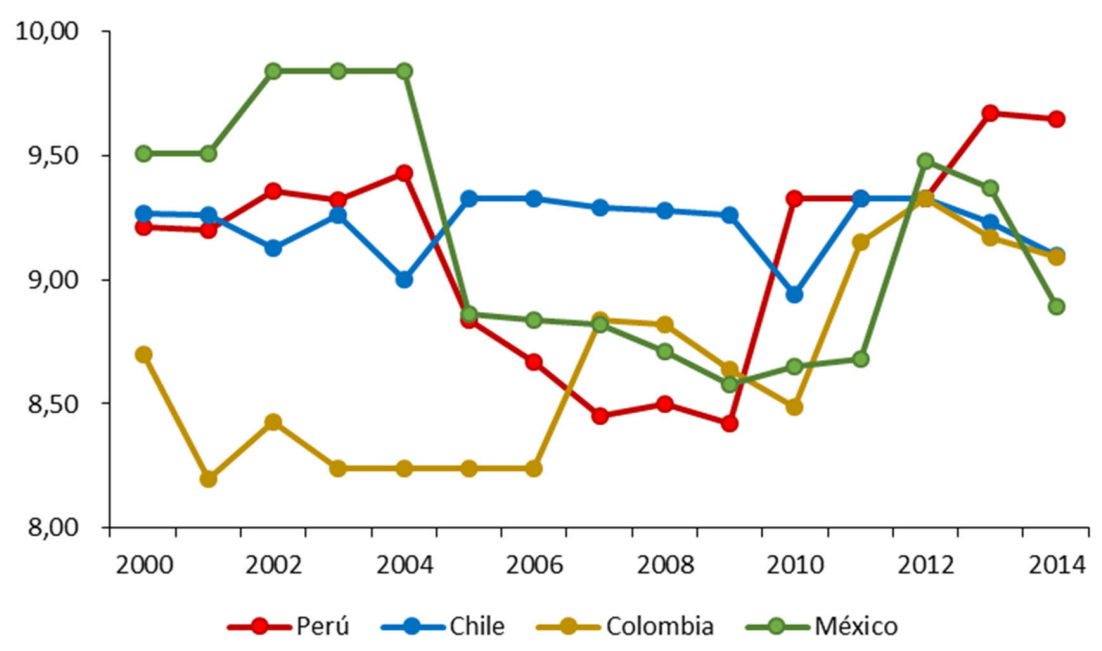

Figura 2. Evolución del índice de libertad en el mercado de créditos en países de la Alianza del Pacífico 2000-2014

Fuente: Fraser Institute, BCRP / Elaboración propia

La correlación de Spearman se muestra en la siguiente tabla:

Tabla 5.

\section{Estadísticos de Spearman}

\begin{tabular}{ccc} 
Periodo & Estadísticos \\
& rs & Prob $>|t|$ \\
\hline $2000-2014$ & 0.27 & 0.32 \\
\hline $2005-2014$ & 0.74 & 0.01 \\
\hline
\end{tabular}

Así, se puede observar que a través del estadístico rs que la relación entre las variables es positiva, tanto para el periodo 2000-2014 como para el 20052014. Sin embargo, en la primera la relación es débil mientras que para la segunda es fuerte pues se acerca a 1. Además, mediante Prob $>|t|$ se rechaza la hipótesis nula de independencia entre las variables, solo para el periodo 2005-2014, por lo que la relación estadística observada para este periodo resulta significativa, mas no para los años 2000-2014.

En la siguiente tabla se detallan los resultados de la estimación del modelo MCO.

Tabla 6.

Estadísticos del MCO

\begin{tabular}{ll}
\multicolumn{1}{c}{ Estadísticos } & \multicolumn{1}{c}{ Valor } \\
Prob $>\mathrm{F}$ & 0.00 \\
\hline $\mathrm{R} 2$ & 0.82 \\
\hline$\beta$ & 107.28 \\
\hline p-value $(\beta)$ & 0.00 \\
\hline$\gamma$ & -119.66 \\
\hline p-value $(\gamma)$ & 0.00 \\
\hline
\end{tabular}

Por lo tanto, el modelo es bueno en términos de bondad de ajuste, pues el $\mathrm{R} 2$ tiende a 1 y a través de la prueba $\mathrm{F}$ (Prob > F) se muestra que la inclusión de las variables exógenas sirven para mejorar el poder explicativo del modelo. Asimismo, $\beta$ nos señala que la relación entre las variables es positiva y estadísticamente significativa 
al $1 \%$ pues tiene un $\mathrm{p}$-value igual a 0 . Por último, $\gamma$ indica que existe una relación negativa y estadísticamente significativa al $1 \%$ entre la endógena y la variable dicotómica de cambio de comportamiento, ello comprueba que efectivamente hay un quiebre durante los años 2000-2004 en relación a todo el periodo de estudio.

\section{DISCUSIÓN}

Del análisis realizado sobre la base de la información del Economic Freedom of The World (EFW), del grado de libertad en el mercado de créditos entre los países de la Alianza del Pacífico, los resultados para el año 2014 son: Perú $(9,65)$, Chile $(9,10)$, Colombia $(9,09)$ y México $(8,89)$. No obstante, el grado de libertad en el mercado de créditos para Perú en el año 2014 (9,65) empeora ligeramente si se compara con el 2013 (9,67), siendo éste el nivel más alto obtenido por nuestro país. Por otro lado, no existen estudios, en donde se muestren la implicancia de la libertad en el mercado de créditos en el stock de créditos otorgados por el sistema financiero al sector privado. En este contexto, el presente estudio resalta la libertad económica en general y la libertad en el mercado de créditos en particular, porque en función a ésta última, los agentes económicos que participan en el sistema financiero, a través del ensayo y error, van adaptando o corrigiendo sus decisiones al momento de participar en las operaciones financieras. Para ello, es vital contar con un gobierno limitado, que no controle las tasas de interés y no establezca barreras regulatorias al mercado de créditos. Es más, cuando se controla las tasas de interés, se afecta de manera indirecta a los clientes financieros, porque hay menos recursos financieros disponibles en el mercado de créditos, y sobre todo, se restringe la libertad de los agentes para participar en dicho mercado. Los altos niveles obtenidos por nues- tro país en el grado de libertad en el mercado de créditos se explican por la no participación del Estado en el sistema financiero. Por el contrario, nuestro sistema se basa en la banca privada para asignar el crédito al sector privado. Siendo así, el esquema actual del sistema financiero peruano se basa en la libre competencia -sin controles de tasas de interés- lo que permite que el mercado asigne eficientemente los recursos desde los ofertantes de fondos (ahorristas) hacia los demandantes de fondos (prestatarios), estimulando así, la canalización del ahorro de los agentes superavitarios hacia el financiamiento de actividades productivas, incidiendo positivamente en el crecimiento económico del país. En virtud que se requieren otros estudios relacionados con presente temática, la agenda de investigación en el futuro debería tratar de responder las siguientes interrogantes: ¿Por qué suceden las crisis de los sistemas financieros?, ¿Cómo influye la interacción de otros componentes de la regulación en el mercado de créditos?, ¿Cuál de los componentes de la libertad en el mercado de créditos explica de mejor manera?

Se concluye de que los datos del grado de libertad en el mercado de créditos permitieron examinar el impacto de la regulación del mercado de créditos sobre el stock de créditos del sistema financiero al sector privado. En tal sentido, la libertad en el mercado de créditos favorece el stock de créditos y consiguientemente, contribuye a profundizar el sistema financiero. No obstante, la mayor libertad en el mercado de créditos es un aspecto esencial para aumentar el stock de créditos pero no es el único elemento.

Se pudo verificar que durante el período 2000-2014, el grado de libertad en el mercado de créditos mejoró para nuestro país, tal como se presentó en la sección de Resultados. El estudio constata que la libertad en el mercado de créditos tiene una participación positiva en el stock de créditos del sistema financiero al sector privado, debido a que añade valor explicativo al modelo que estima la relación.

El estudio constata que el grado de libertad en el mercado de créditos entre los países de la Alianza del $\mathrm{Pa}$ cífico (AP) tiene un similar comportamiento, denotando una estrecha relación entre los mismos. En cuanto al último año, si bien no se observa la misma tendencia, Perú es el país que más crece en cuanto al índice de regulación del mercado de créditos, explicado fundamentalmente por la libre competencia del sistema financiero.

La investigación establece las bases para futuros estudios acerca de las causas que generan las crisis de los sistemas financieros y la influencia de la interacción de otros componentes de la libertad en el mercado de créditos en el stock de créditos del sistema financiero al sector privado.

\section{REFERENCIAS BIBLIOGRÁFICAS}

Akerlof, G. \& Shiller, R. (2014). Animal Spirits. Madrid: Unión Editorial S.A.

Cameron, C., \& Trivedi, P. (2009). Microeconometrics using Stata. Texas: Stata Press Publication.

Choy, M., Costa, E. \& Churata, E. (2015). Radiografía del costo del crédi-to en el Perú - Serie de Documentos de Trabajo. Lima: Banco Central de Reserva del Perú.

Gwartney, J., Lawson R., \& Hall J. (2016). Economic Freedom of the World 2016 Annual Report. Vancouver: Fraser Institute.

Hayek, F. (2014). Derecho legislación y libertad. Madrid: Unión Editorial S.A. 
Rodríguez, V. (2016). Libertad económica y bienestar individual en Perú. Quipukamayoc. 24(46), pp. 25 y 36.

Rodríguez, V. (2015). Principios ge-nerales de una sociedad libre para fortalecer el orden espontáneo del mercado. Quipukamayoc. 23(44), pp. 125 - 138.
Rodríguez, V. (2014). Régimen financiero de la Constitución de 1993. Quipukamayoc. 22(42), pp. 147 y 162.

North, D. (1993). Instituciones, cambio institucional y desempeño económico. México D.F.: Fondo de Cultura Económica.
Mises, L. (2012). Teoría del dinero y del crédito. Madrid: Unión Editorial S.A.

Varian, H. (2006). Microeconomía Intermedia. Barcelona: Antoni Bosch, editor S.A. 